NASA Contractor Report 195015

ICASE Report No. 94-96
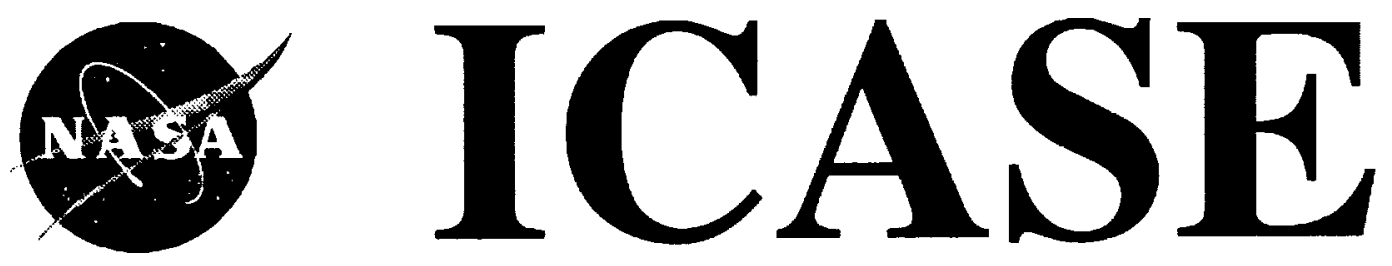

\title{
AN ALGORITHM FOR SOLVING THE SYSTEM-LEVEL PROBLEM IN MULTILEVEL OPTIMIZATION
}

\section{R. J. Balling}

\section{J. Sobieszczanski-Sobieski}

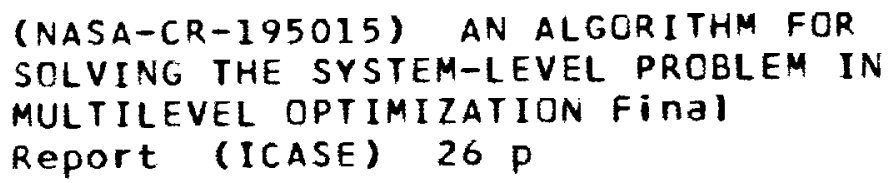

Institute for Computer Applications in Science and Engineering NASA Langley Research Center Hampton, VA 23681-0001 
$7 \cdot 7$ 


\title{
AN ALGORITHM FOR SOLVING THE SYSTEM-LEVEL PROBLEM IN MULTILEVEL OPTIMIZATION*
}

\author{
R. J. Balling \\ Brigham Young University \\ Provo, UT 84602 \\ J. Sobieszczanski-Sobieski \\ NASA Langley Research Center \\ Hampton, VA 23681-0001
}

\begin{abstract}
A multilevel optimization approach which is applicable to nonhierarchic coupled systems is presented. The approach includes a general treatment of design (or behavior) constraints and coupling constraints at the discipline level through the use of norms. Three different types of norms are examined-the max norm, the Kreisselmeier-Steinhauser (KS) norm, and the $1_{p}$ norm. The max norm is recommended. The approach is demonstrated on a class of hub frame structures which simulate multidisciplinary systems. The max norm is shown to produce system-level constraint functions which are non-smooth. A cutting-plane algorithm is presented which adequately deals with the resulting corners in the constraint functions. The algorithm is tested on hub frames with increasing number of members (which simulate disciplines), and the results are summarized.
\end{abstract}

\footnotetext{
*This research was supported by the National Aeronautics and Space Administration under NASA Contract No. NAS1-19480 while the first author was in residence at the Institute for Computer Applications in Science and Engineering (ICASE), NASA Langley Research Center, Hampton, VA 23681-0001.
} 
$\longdiv { 1 }$ 


\section{Introduction}

This paper is concerned with the optimization of systems whose mathematical model is an assembly of coupled modules, each transforming input to output. Analyses and optimizations, usually corresponding to engineering disciplines or physical components, may be executed within these modules. The term "discipline" will be used throughout to mean such a module.

Approaches to this problem can be divided into single-level optimization approaches and multilevel optimization approaches (Balling and Sobieszczanski-Sobieski 1994). In the former, only a single optimization problem is solved for the entire system, while in the latter, optimization problems are solved within the disciplines as well as for the system as a whole. Thus, in a two-level optimization approach, there is an optimization at the system level, and there are optimizations at the discipline level. It may be possible to view a discipline as a system itself composed of subdisciplines. It this case, a three-level optimization approach may be employed wherein optimizations occur at the system-level, the disciplinelevel, and the subdiscipline-level. This scheme is readily extendable beyond three levels. The focus of this paper is on multilevel optimization, although single-level optimization is used for comparison.

Over a decade ago, a method for linearly decomposing a single large optimization problem into multilevel optimization problems was suggested (Sobieszczanski-Sobieski 1982). In that method, the system was decomposed into disciplines, and optimizations and analyses were performed at the system level and within each of the disciplines. The disciplines were coupled to the system but not to each other. Such a system was referred to as a "bierarchic system" as shown in Figure 1. The discipline-level optimization problems sought to minimize violation in the design (or behavior) constraints while satisfying equality constraints on the coupling variables passed from the system to the discipline. The need to satisfy equality constraints was identified as a source of numerical difficulties that occasionally arose in applications (Thareja and Haftka 1986). In alternative formulations, the discipline-level optimization problems seek to minimize discrepancy in the coupling equations while satisfying the design (or behavior) constraints (Schmit and Ramanathan 1973; Sobieszczanski-Sobieski 1993).

The first objective of this paper is to present a more general multilevel optimization approach than the approaches suggested in Sobieszczanski-Sobieski 1993. The multilevel optimization approach presented here has been extended to nonhierarchic multidisciplinary systems. In nonhierarchic systems, all disciplines are on the same level, and analysis and optimization associated with the coordination of the system is implied (see Figure 1). In nonhierarchic systems, each discipline may be coupled to every other discipline. The traditional hierarchic system may be viewed as a nonhierarchic system by treating the "system" as a discipline on the same level as the other disciplines (see Figure 1). The multilevel optimization approach presented here also generalizes the treatment of the design constraints and coupling equations at the discipline-level. The discipline-level optimization problem is formulated as the minimization of a norm of both design constraint violation and discrepancy in the coupling equations. Such a formulation guarantees that a feasible solution always exists for the discipline-level optimization problem. Three different norms will be examined---the max norm, the KS norm (Kreisselmeier and Steinhauser 1983), and the $l_{p}$ norm. 
The second objective of this paper is to present an algorithm for efficiently solving the system-level optimization problem. It will be demonstrated that when the max norm is used in the discipline-level optimization problems, the system-level optimization problem posșesses constraint functions which are non-smooth. A cutting-plane algorithm embedded within a move-limit strategy will be presented which adequately treats non-smooth functions. Results will be presented for test problems of increasing size.

The paper begins by presenting the general single-level and multilevel optimization approaches for nonhierarchic systems. The calculation of sensitivities for the approaches will be discussed. The approaches will then be demonstrated on an example of a hub frame which was selected because the data flow in the analysis and optimization of a structure composed of substructures or finite elements is a good analog of the data flow in the analysis and optimization of a system composed of coupled disciplines. The paper will then use this example to examine the three norms for the discipline-level optimization problem. The cutting-plane algorithm and move-limit strategy for the system-level optimization problem will be presented, and numerical results will be discussed.

\section{Single and Multilevel Optimization Approaches}

Consider the three-discipline coupled system shown in Figure 2. Each discipline in this system has an associated analysis program which computes output values of the functions from input values of the variables. A three-discipline system was chosen as a basis for discussion because it is small enough to keep the discussion simple but large enough to see a general pattern.

The system is nonhierarchic because each discipline is coupled to every other discipline, and no discipline is viewed as being "above" the others. The vectors $y_{12}, y_{13}, y_{21}, y_{23}, y_{31}$, and $y_{32}$ are the coupling functions. Note that $y_{i j}$ contains those functions computed in Discipline $i$ which are needed as input to Discipline $\mathrm{j}$. It is these coupling functions which complicate the order of execution of the disciplinary analyses. By associating with each vector of coupling functions a corresponding vector of coupling variables $\left(\mathrm{y}_{12}{ }^{*}, \mathrm{y}_{13}{ }^{*}, \mathrm{y}_{21}{ }^{*}, \mathrm{y}_{23}{ }^{*}, \mathrm{y}_{31}{ }^{*}\right.$, and $\left.\mathrm{y}_{32}{ }^{*}\right)$, the disciplinary analyses may be executed in parallel. Each receives coupling variables as input and computes coupling functions as output. One of the tasks of the single-level or multilevel optimization approach is to satisfy coupling constraints which enforce equality between each coupling variable and its corresponding coupling function.

The vectors $x, x_{1}, x_{2}$, and $x_{3}$ are mutually exclusive sets of design variables needed as input to the analyses. Note that $x$ contains system design variables needed by more than one discipline, while the vectors $x_{1}, x_{2}$, and $x_{3}$ contain disciplinary design variables needed by Disciplines 1,2 , and 3 , respectively. The vectors $g_{1}, g_{2}$, and $g_{3}$ contain the design constraint functions. These represent the constraints which guard against unacceptable behavior. Only inequality constraints are considered here, and it is assumed that each constraint has been formulated such that zero is its allowable value, and it is satisfied when less than zero. The vectors $f_{1}, f_{2}$, and $f_{3}$ contain the design objective functions. These represent objectives such as the maximization of benefits and the minimization of costs. It is assumed that each objective has been formulated such that it is improved through minimization, and the value of zero is associated with a selected target value. 
The optimization problem may be solved on a single level as follows:

\section{Single-Level Optimization Problem}

Find:

$$
\mathrm{f}, \mathrm{x}, \mathrm{x}_{1}, \mathrm{x}_{2}, \mathrm{x}_{3}, \mathrm{y}_{12}{ }^{*}, \mathrm{y}_{13}{ }^{*}, \mathrm{y}_{21}{ }^{*}, \mathrm{y}_{23}{ }^{*}, \mathrm{y}_{31}{ }^{*}, \mathrm{y}_{32}{ }^{*}
$$

Minimize: $\quad \mathbf{f}$

$\begin{array}{llll}\text { Satisfy: } & \mathrm{g}_{1}<0, & \mathrm{~g}_{2}<0, & \mathrm{~g}_{3}<0, \\ & \mathrm{f}_{1}<\mathrm{f}, & \mathrm{f}_{2}<\mathrm{f}, & \mathrm{f}_{3}<\mathrm{f}, \\ & \mathrm{y}_{12}=\mathrm{y}_{12}{ }^{*}, & \mathrm{y}_{13}=\mathrm{y}_{13}{ }^{*}, & \\ \mathrm{y}_{21}=\mathrm{y}_{21}{ }^{*}, & \mathrm{y}_{23}=\mathrm{y}_{23}{ }^{*}, & \\ \mathrm{y}_{31}=\mathrm{y}_{31}{ }^{*}, & \mathrm{y}_{32}=\mathrm{y}_{32}{ }^{*} & \end{array}$

Note the addition of the scalar design variable, $f$, the system objective. By minimizing this variable and constraining it to be greater than each of the design objectives, the maximum of the objectives is effectively minimized. This "minimax" formulation is just one of several ways for treating paretooptimization problems. A reminder should be made that the optimal results will be affected by the scaling and the choice of allowable/target values for the design constraint and objective functions. The minimax formulation essentially transforms design objectives into design constraints.

The single-level optimization problem may be solved directly (Haftka et al. 1992), or it may force satisfaction of the coupling constraints at each optimization iteration (Grossman et al. 1989; Hajela et al. 1990; Sobieszczanski-Sobieski et al. 1991; Haftka et al. 1992). The coupling constraints may be satisfied via Newton's method or via a nongradient fixed-point iteration method.

The optimization problem may also be solved on two levels: the system level and the discipline level. In this multilevel approach, the system-level optimization problem is:

\section{System-Level Optimization Problem}

Find:

$$
\mathrm{f}, \mathrm{x}, \mathrm{y}_{12}{ }^{*}, \mathrm{y}_{13}{ }^{*}, \mathrm{y}_{21}{ }^{*}, \mathrm{y}_{23}{ }^{*}, \mathrm{y}_{31}{ }^{*}, \mathrm{y}_{32}{ }^{*}
$$

Minimize: $\quad$ f

Satisfy: $\quad d_{1}<0, \quad d_{2}<0, \quad d_{3}<0$

The scalars $d_{1}, d_{2}$, and $d_{3}$ are discrepancy functions which are computed by solving the discipline-level optimization problems which can be formulated using three different norms: the max norm, the KS norm, and the $l_{p}$ norm. The discipline-level optimization problem for Discipline $i$ using the max norm is: 


\section{Discipline-Level Optimization Problem (Max Norm)}

Find: $\quad x_{i}, d_{i}$

Minimize: $\quad d_{i}$

Satisfy: $\quad g_{\mathrm{i}}<\mathrm{d}_{\mathrm{i}}$,

$$
\mathrm{f}_{\mathbf{i}}-\mathrm{f}<\mathrm{d}_{\mathrm{i}} \text {, }
$$

$y_{i j}-y_{i j}^{*}<d_{i}, \quad$ for $j \neq i$

$\mathrm{y}_{\mathrm{ij}}^{*}-\mathrm{y}_{\mathrm{ij}}<\mathrm{d}_{\mathrm{i}}$

for $j \neq i$

Note that discipline-level optimization seeks to minimize the max norm of the violation in the design constraints (1), (2) and the coupling constraints (3), (4). The system design variables and the coupling variables are treated as fixed parameters during discipline-level optimization. The optimum value of the discrepancy function is the only function which is sent back from discipline-level optimization to systemlevel optimization.

An alternative to the max norm in discipline-level optimization is possible which uses the KS family of norms. For Discipline i:

\section{Discipline-Level Optimization Problem (KS Norm)}

Find: $\quad \mathbf{x}_{\mathbf{i}}$

Minimize: $\quad d_{i}=(1 / \rho) \ln \left\{\Sigma \exp \left(\rho g_{i}\right)+\Sigma \exp \left(\rho\left(f_{i}-f\right)\right)+(1 / 2) \sum_{j=i}\left[\exp \left(\rho\left(y_{i j}-y_{i j}{ }^{*}\right)\right)+\Sigma \exp \left(\rho\left(y_{i j}{ }^{*}-y_{i j}\right)\right)\right]\right\}$

The parameter $\rho$ is a positive real number, the sum $\sum_{j \neq i}$ is over disciplines other than Discipline $i$, and the other sums are over the elements of the vectors involved. As $\rho$ goes to infinity, the KS norm becomes equivalent to the max norm. Otherwise, the KS norm is greater than the max norm by an amount which is bounded by $(1 / \rho) \ln (m)$ where $m$ is the sum of the sizes of the vectors $g_{i}, f_{i}$, and $y_{i j}$ for $j \neq i$.

Another family of norms that is possible for discipline-level optimization is the $l_{p}$ family. For Discipline i:

Discipline-Level Optimization Problem ( $l_{p}$ Norm)

Find: $\quad \mathbf{x}_{\mathbf{i}}$

Minimize: $\quad \mathrm{d}_{\mathrm{i}}=\left\{\Sigma\left(\max \left(0, \mathrm{~g}_{\mathrm{i}}\right)\right)^{\mathrm{p}}+\Sigma\left(\max \left(0, \mathrm{f}_{\mathrm{i}}-\mathrm{f}\right)\right)^{\mathrm{p}}+\sum_{\mathrm{j} \times \mathrm{i}}\left[\Sigma\left|\mathrm{y}_{\mathrm{ij}}-\mathrm{y}_{\mathrm{ij}}{ }^{*}\right|^{\mathrm{p}}\right]\right\}^{1 / \mathrm{p}}$ 
The parameter $\mathrm{p}$ is a positive integer and the sums are the same as for the KS family of norms. The $l_{2}$ norm is the familiar Euclidean norm, and as $p$ goes to infinity, the $l_{p}$ norm becomes equivalent to the $\max$ norm.

\section{Sensitivity Analysis}

Since the optimization algorithms used to solve the single-level, system-level, and discipline-level optimization problems are gradient-based, it is necessary to compute derivatives of the constraints and objectives with respect to the variables in each optimization problem.

Derivatives of outputs with respect to inputs for Analyses 1, 2, and 3 may either be approximated by finite differences or analytically calculated as part of the analysis itself. The latter approach yields more accurate derivatives, and may be more computationally efficient.

In the case of multilevel optimization, the discipline-level optimizations are viewed from the system level as black boxes, and their sole purpose is the calculation of the discrepancy functions and their sensitivities. For example, Discipline $i$ must compute $d_{i}$ through discipline-level optimization and derivatives of the optimum $d_{i}$ with respect to $y_{j i}{ }^{*}$ and $y_{i j}{ }^{*}$ (for $j \neq i$ ). These optimum sensitivities can be calculated directly from the Lagrange multipliers of the discipline-level optimization and the sensitivities computed by Analysis i (Barthelemy and Sobieszczanski-Sobieski 1983). Specifically, if the max norm formulation is used for Discipline $\mathrm{i}$ :

$$
\begin{aligned}
& \mathrm{dd} / \mathrm{d}\left(\mathrm{y}_{\mathrm{ji}}{ }^{*}\right)_{\mathrm{k}}=\Sigma_{\mathrm{l}}(\lambda)_{1} \partial\left(\mathrm{g}_{\mathrm{i}}\right)_{l} / \partial\left(\mathrm{y}_{\mathrm{ji}}{ }^{*}\right)_{\mathrm{k}}+\Sigma_{\mathrm{l}}(\gamma)_{1} \partial\left(\mathrm{f}_{\mathrm{i}}\right)_{\mathrm{l}} / \partial\left(\mathrm{y}_{\mathrm{ji}}{ }^{*}\right)_{\mathrm{k}}+\Sigma_{1}(\mu-v)_{1} \partial\left(\mathrm{y}_{\mathrm{ij}}\right)_{\mathrm{l}} / \partial\left(\mathrm{y}_{\mathrm{ji}}{ }^{*}\right)_{\mathrm{k}} \quad \text { for } \mathrm{j} \neq \mathrm{i} \\
& \mathrm{dd}_{\mathrm{i}} / \mathrm{d}\left(\mathrm{y}_{\mathrm{ij}}{ }^{*}\right)_{\mathrm{k}}=(v-\mu)_{\mathrm{k}} \quad \text { for } \mathrm{j} \neq \mathrm{i}
\end{aligned}
$$

where the subscripts outside the parentheses refer to elements of the vectors within the parentheses. The vectors $\lambda, \gamma, \mu$, and $v$ contain the Lagrange multipliers of constraints (1), (2), (3), and (4), respectively.

\section{Application to Hub Frames}

Consider the two-member hub frame of Figure 3. It is the simplest case of a hub frame whose members extend radially from Node $P$ like spokes from a hub. The loads for each loading case, the material properties, and the nodal coordinates are fixed, and it is desired to find the optimum cross-sectional dimensions of the two members. Volume is to be minimized, and design constraints are imposed on the displacements at Node $P$ as well as on stress and buckling in the members. 
Considering the structure as an analog of a multidisciplinary system, the two-member hub may be treated as a system composed of three disciplines:

Discipline 1: frame analysis of the entire hub

Discipline 2: analysis of Member PQ

Discipline 3: analysis of Member PR

Table 1 indicates how each of the vectors of variables and functions defined for the general threediscipline system of Figure 2 may be specialized for this specific example. In this table, N, M, and V are the axial force, bending moment, and shear force, respectively, at End P of the member, and $A$ and $I$ are the area and moment of inertia of the member. The displacement constraints at Node $\mathrm{P}$ for each loading case consist of constraints on the resultant translational displacement and on the rotational displacement. There are nine stress constraints for each loading case for each member at locations throughout the crosssection at both ends of the member. There are nine buckling constraints for each loading case for each member including in-plane and out-of-plane buckling of the member as a whole as well as local buckling of the flanges and the web. Computational details of these constraints are given in the Appendix.

The analysis for Discipline 1 (Analysis 1 ) receives A's and I's as input and computes the frame volume, the displacements at Node P, and the N's, M's, and V's for the members. Analyses 2 and 3 receive their N's, M's, V's, and b's, t's, and h's as input and compute their A's, I's, and stress and buckling constraints.

The traditional approach to frame optimization is a single-level optimization approach where the coupling constraints are implicitly solved for the coupling variables at each optimization iteration. Solving the coupling constraints can be accomplished without iteration in the case of frame optimization because although the N's, M's, and V's are functions of the A's and I's, the A's and I's are not functions of the N's, M's, and V's. Thus, at each optimization iteration, a portion of Analyses 2 and 3 is executed to compute $y_{21}$ and $y_{31}$, then Analysis 1 is executed to compute $y_{12}, y_{13}, f_{1}$, and $g_{1}$, and finally the remainder of Analyses 2 and 3 is executed to compute $g_{2}$ and $g_{3}$. With the coupling variables and coupling constraints eliminated in this fashion, the single-level optimization problem for the two-member hub becomes:

\section{Single-Level Optimization Problem}

Find: $\quad \mathbf{x}_{2}, \mathbf{x}_{3}$

Minimize: $\quad f_{1}$

Satisfy: $\quad \mathrm{g}_{1}<0, \quad \mathrm{~g}_{2}<0, \quad \mathrm{~g}_{3}<0$ 
Note that since this problem has a single objective which is computed in Discipline 1 , one may take $\mathrm{f}=\mathrm{f}_{1}$.

The multilevel optimization approach to the hub problem includes system-level optimization and discipline-level optimization for Disciplines 2 and 3. There is no discipline-level optimization for Discipline 1 since this discipline has no design variables to be optimized. It is assumed that Analysis 1 is always executed sequentially before the optimizations of Disciplines 2 and 3. This allows elimination of the coupling variables $\mathrm{y}_{12}{ }^{*}$ and $\mathrm{y}_{13}{ }^{*}$ since the coupling functions $\mathrm{y}_{12}$ and $\mathrm{y}_{13}$ are available for input directly into Analyses 2 and 3. The system-level optimization problem is:

\section{System-Level Optimization Problem}

Find: $\quad \mathrm{y}_{21}{ }^{*}, \mathrm{y}_{31}{ }^{*}$

Minimize: $\quad \mathbf{f}_{1}$

Satisfy: $\quad \mathrm{g}_{1}<0, \quad \mathrm{~d}_{2}<0, \quad \mathrm{~d}_{3}<0$

At each iteration of system-level optimization, Analysis 1 is executed to compute $f_{1}, g_{1}, y_{12}$, and $y_{13}$, and then the optimization problems for Disciplines 2 and 3 are solved to compute $d_{2}$ and $d_{3}$. The discipline-level optimization problem for Discipline 2 (similar for Discipline 3) is:

\section{Discipline-Level Optimization Problem (Max Norm)}

Find: $\quad \mathbf{x}_{2}, \mathbf{d}_{2}$

Minimize: $\quad \mathrm{d}_{2}$

Satisfy: $\quad g_{2}<d_{2}$,

$$
\begin{aligned}
& y_{21}-y_{21}{ }^{*}<d_{2}, \\
& y_{21}{ }^{*}-y_{21}<d_{2}
\end{aligned}
$$

Discipline 2 receives $\mathrm{y}_{12}$ and $\mathrm{y}_{21}{ }^{*}$ from the system level which are held fixed during discipline-level optimization. At each iteration of discipline-level optimization for Discipline 2, Analysis 2 is executed to compute $g_{2}$ and $y_{21}$.

Discipline-level optimization can also be formulated for the hub problem using the $\mathrm{KS}$ and the $\mathrm{l}_{\mathrm{p}}$ norms. For Discipline 2 (similar for Discipline 3): 


\section{Discipline-Level Optimization Problem (KS Norm)}

Find: $\quad \mathbf{x}_{2}$

Minimize: $\quad d_{2}=(1 / \rho) \ln \left\{\operatorname{\Sigma exp}\left(\rho g_{2}\right)+(1 / 2)\left[\operatorname{Lexp}\left(\rho\left(y_{21}-y_{21}{ }^{*}\right)\right)+\Sigma \exp \left(\rho\left(y_{21}{ }^{*}-y_{21}\right)\right)\right]\right\}$

\section{Discipline-Level Optimization Problem $\left(l_{p}\right.$ Norm)}

Find: $\quad \mathbf{x}_{2}$

Minimize: $\quad \mathrm{d}_{2}=\left\{\Sigma\left(\max \left(0, \mathrm{~g}_{2}\right)\right)^{\mathrm{p}}+\Sigma\left|\mathrm{y}_{21}-\mathrm{y}_{21} *\right|^{\mathrm{p}}\right\}^{1 / \mathrm{p}}$

The single-level, system-level, and discipline-level optimization problems are easily formulated for hub frames with more members such as shown in Figures 4 and 5 . Note that the frame analysis for a several-member hub is as simple as the frame analysis for a two-member hub since there are only three displacement degrees of freedom in either case. Thus, hub frames provide a convenient tool for studying the effect of increasing the size of the system by increasing the number of members without increasing the system analysis dimensionality.

\section{Plots of the Discrepancy Function}

The single-level optimization problem and the discipline-level optimization problems are well-posed problems and may be solved by standard nonlinear programming (NLP) algorithms. However, the systemlevel optimization problem is a non-smooth optimization problem. Figure 6 shows contour plots of the discrepancy function for Member $\mathrm{PQ}$ of the two-member hub as it looks from the system level. The two axes are the elements of the $y_{21}{ }^{*}$ vector (i.e., the $A$ and $I$ for member PQ). The elements of the $y_{31}{ }^{*}$ vector (i.e., the $A$ and I for member PR) were held fixed in these plots. Data for the plots were generated by dividing the plotted region into a $21 \times 21$ point rectangular mesh and computing $d_{2}$ at each mesh point. The computation of $d_{2}$ at each mesh point required a frame analysis followed by a discipline-level optimization for Member PQ. The discipline-level optimizations employed the three different norms as indicated for the three different plots.

The large upper right region of the plot for the max norm has a constant value of zero. This region may be regarded as the conservative region. Here the values in the $y_{21}{ }^{*}$ vector sent down from the system level are large enough that the discipline-level optimization is able to completely satisfy both the design and coupling constraints. Specifically, this means that the discipline-level optimization was able to find values for the b's, $t$ 's, and h's for Member $P Q$ which satisfied all stress and buckling constraints as well as matched the $\mathrm{A}$ and $\mathrm{I}$ sent down from the system. This was not possible for the region on the left side and on the bottom of the plot which are regarded as the unconservative region. In this region, violation 
remains in either the design constraints or the coupling constraints or both, and the maximum value of that violation is positive.

Note also the sharp comers in the contours at the lower left portion of the plot for the max norm. These comers occur when there is a change in the set of active constraints at the optimum of the discipline-level optimization problem. The set of active constraints are those design and coupling constraints which control the max norm. Thus, on one side of the comer, one set of constraints is maximum, while on the other side, another set of constraints is maximum. In the case of the two-member hub, the optimum to the system-level optimization problem lies at the intersection of the locus of these corners with the conservative region.

One would expect these phenomena to be observed in multilevel optimization problems in general. Specifically, one would expect the existence of a conservative region where all design and coupling constraints can be satisfied. One would also expect corners in the discrepancy function in the unconservative region as changes occur in the set of active constraints in the discipline-level optimum. Furthermore, it is likely that the system-level optimum lies at such comers on the boundary of the conservative region.

Note that the corners for the KS norm are rounded. Note also that the spacing of the contours increases as one moves from the unconservative region to the conservative region. In fact, there is no clear boundary between the two regions. The value of discrepancy in the conservative region is asymptotic to a positive value equal to $(1 / \rho) \ln$ (number of active constraints). Since the number of active constraints in the conservative region is not known a priori, this asymptotic value is difficult to estimate. In the system-level optimization problem, discrepancies must be constrained to be less than some positive $\varepsilon$ which is greater than this asymptotic value rather than be constrained to be less than zero. To converge to the true optimum, one must let $\rho$ go to infinity and $\varepsilon$ go to zero during the system optimization process. A drawback to using the KS norm is the difficulty in selecting appropriate values for $\rho$ and $\varepsilon$ throughout the optimization process. Another disadvantage of the $\mathrm{KS}$ norm is that although it smoothes out the corners in the discrepancy function, it does so by increasing nonlinearity. This will increase the computational effort to solve the system optimization problem.

The contour plot for the $\mathrm{l}_{2}$ norm is very similar to that of the max norm. Although the corner is sharp at the boundary of the conservative region, it becomes rounder as one moves further into the unconservative region. Two characteristics make the use of the $l_{2}$ norm undesirable. First, solution time for discipline-level optimization using standard NLP software is noticeably longer than when the max norm is used---almost an order of magnitude longer for hub frames. Second, the results of discipline-level optimization appear to be noisy along the left side of the conservative region. Both the increased solution time and the noise arise from the fact that the $l_{2}$ objective function in the discipline-level optimization problem is relatively flat near the optimum. This makes it difficult for standard NLP algorithms to converge to the optimum even when tight convergence tolerances are imposed.

Therefore, it is recommended that the max norm be used in multilevel optimization. It will be assumed that the max norm is used throughout the remainder of this paper. 


\section{System-Level Optimization Algorithm}

The algorithm for solving the system-level optimization problem should exploit the fact that sensitivities of the discrepancy functions can be obtained at virtually no extra cost beyond calculating the discrepancy functions themselves as indicated in Section 3. Therefore, it is recommended that the system-level optimization algorithm consist of the successive solution of a series of approximate optimization problems. In each such optimization problem, linear approximations of the discrepancy functions are constructed from the values and sensitivities computed at the current design.

The system-level optimization algorithm just described is basically the Sequential Linear Programming (SLP) algorithm. A major difficulty with using SLP to solve the system-level optimization problem arises from the existence of corners in the discrepancy functions. These comers may cause oscillatory behavior in the SLP algorithm as solutions to the successive linear programming (LP) problems jump back and forth from one side of a corner to the other. Oscillatory behavior is also observed when other NLP algorithms are used which determine a gradient-based search direction followed by a line search since search directions are based on information from only one side of the corner. The oscillatory behavior in SLP may be circumvented to some extent by imposing tight move limits so that the current design can carefully "creep up" on such corners, but this may require solution of a prohibitively large number of LP problems.

A truly non-smooth optimization algorithm is needed. One can modify the basic SLP algorithm so that it uses gradient information on both sides of corners by simply retaining the linearizations of the discrepancy functions from the previous LP problems. Thus, the number of constraints grows from one LP problem to the next as the linear approximation of each $d_{i}<0$ at the current design is appended to the linear approximations of the same at previous designs. Thus, each of the non-smooth constraints $d_{i}<0$ is approximated by cutting planes from all prior LP problems. The smooth design constraints from Discipline $1\left(g_{1}<0\right)$ on the displacements at Node $P$ may also be approximated by cutting planes from all prior LP problems. This algorithm is a form of Kelley's Cutting Plane (KCP) algorithm (Kelley 1960). The steps for the KCP algorithm applied to the two-member hub are shown in the inner box of Figure 7.

Since the contours of the discrepancy function for the max norm shown in Figure 6 appear to be nearly piecewise linear, one might expect the KCP algorithm to be quite efficient for the hub frame. It has also been observed in the field of structural optimization that linear approximations of displacement functions are reasonable. The KCP algorithm will converge to the optimum of the non-smooth systemlevel optimization problem if all of the discrepancy and displacement functions are convex. If any of these functions are concave, the KCP algorithm may:

Case \#1) converge to a feasible, though suboptimal, solution;

Case \#2) encounter an LP problem for which there is no feasible solution.

The KCP algorithm may be embedded in a move limit strategy as shown in Figure 7 to mitigate the above two problems. Initially, large move limit ranges are assumed and the $\mathrm{KCP}$ algorithm is executed. 
If normal convergence occurs (Case \#1) for this first cycle, the move limit ranges are centered about the new optimum, and the move limit ranges are reduced for those variables which were not pushed up against the previous move limit bounds. All previous linear approximations are then thrown out and the KCP algorithm is executed again for the next cycle. If at any cycle the KCP algorithm encounters an LP problem with no feasible solution (Case \#2), one must return to the feasible design at the start of the cycle, reduce the move limit ranges, throw out all previous linear approximations, and execute the KCP algorithm again. If this happens in the first cycle and the starting design is infeasible, a feasible (or more nearly feasible) starting design must be found by some other means such as suggested in (Barthelemy and Riley 1988). Since the move limit strategy is basically heuristic, designer expertise should be consulted in selecting starting and reduced move limit ranges.

\section{Results}

The multilevel approach using the KCP algorithm to solve the system-level optimization problem was applied to the two-member hub to solve the following two problems:

Problem A: constraints on displacements at Node P neglected

Problem B: constraints on displacements at Node P considered

Results are plotted in Figure 8 for two different starting designs---a conservative feasible starting design and an infeasible starting design. The normalized frame volume is plotted versus iterations of the $\mathrm{KCP}$ algorithm. The frame volume was normalized by dividing by the optimum frame volume as computed from single-level optimization. A sequential quadratic programming algorithm (SQP) was used to solve both the single-level optimization problem and the discipline-level optimization problems.

Note that the KCP algorithm reached the optimum after 4-7 iterations. Additional move limit cycles were not necessary for the two-member hub since the optimum was achieved in one cycle. The design after the first iteration was quite infeasible and had a very low frame volume. The optimum design was then approached from the infeasible side. There are four optimization variables for the system-level optimization problem, namely: $\mathrm{y}_{21}{ }^{*}=\{\mathrm{A}, \mathrm{I}$ for member $\mathrm{PQ}\}$ and $\mathrm{y}_{31}{ }^{*}=\{\mathrm{A}, \mathrm{I}$ for member $\mathrm{PR}\}$. The optimum solutions for both Problems A and B had four controlling cutting planes. For Problem A, the optimum lies at the intersection of two cutting planes from each of the constraints $d_{2}<0$ and $d_{3}<0$. For Problem $B$, the optimum lies at the intersection of one cutting plane from each of the constraints $d_{2}<0$ and $d_{3}<0$ and two cutting planes from the translational displacement constraint of Load Case 1.

Problems A and B were solved starting from a conservative feasible design and an infeasible design for the eight-member hub shown in Figure 4 and the twenty-member hub shown in Figure 5. Results are plotted in Figure 9 and Figure 10, respectively. Again, the frame volume at each iteration is normalized by dividing by the optimum frame volume achieved by single-level optimization. Three move-limit cycles are shown for the eight-member hub and six move-limit cycles are shown for the twenty-member hub. 
Additional cycles produced little change in the final design. Move limit ranges were reduced by a factor of two from cycle to cycle.

In each cycle, the KCP algorithm drops to a low-volume infeasible design within the move limits and works its way back to a feasible design at the end of the cycle. Note that the optimum design was not necessarily achieved after one cycle in these problems indicating a degree of concavity in at least some of the constraint functions. For the eight-member hub, the designs at the end of the cycles form a sequence of feasible designs whose frame volumes monotonically decrease to the optimum. However, the frame volume of the sequence of feasible designs is not monotonically decreasing for the twenty-member hub. In fact, additional cycles for Problem $\mathrm{A}$ of the twenty-member hub did not produce further improvement. The lack of monotonicity and the convergence to a value of the objective somewhat higher than the minimum for the twenty-member hub underscore the fact that the move limit strategy is a heuristic procedure rather than a formal optimization algorithm.

It is interesting that the number of iterations per cycle does not appear to increase with number of disciplines (members) but remains around ten. This means that for hub frames at least, ten frame analyses and ten discipline-level optimizations yield a fairly good feasible design. Each cycle thereafter requires an investment of ten more analyses and discipline-level optimizations to produce feasible designs with generally improving frame volume.

It was noted that for the two-member hub, the computational time for conventional single-level optimization was faster than the total time of multilevel optimization. For the eight-member hub, the computational times were roughly the same. For the twenty-member hub, the computational time for single-level optimization was significantly longer than the total time of ten cycles of multilevel optimization. This can be explained from the fact that hub frames are optimization-intensive rather than analysis-intensive. The analysis of a hub frame consists of solving a $3 \times 3$ set of linear stiffness equations regardless of number of members. However, the single-level optimization problem involves:

eight-member hub

48 variables

292 constraints twenty-member hub

120 variables

724 constraints

Multilevel approaches break these large optimization problems into smaller ones. Since typical NLP algorithms involve direct equation solvers, one may solve several smaller optimization problems faster than a single large problem. The discipline-level optimization problem for each member involves 6 variables and 40 constraints, and the system-level optimization problem involves:

eight-member hub

16 variables

12 constraints twenty-member hub

40 variables

24 constraints 
It should be noted that no attempt was made to exploit the opportunity for concurrent optimization of the disciplines. That opportunity is intrinsic in the multilevel approach but absent in the single-level approach. It generally alters the elapsed time comparisons in favor of the multilevel approach.

\section{Conclusions}

A multilevel optimization approach was formulated for nonhierarchic multidisciplinary systems and specialized for hub frame structures. The discipline-level optimization problems were formulated as the minimization of a norm of both coupling constraint and design constraint violation, and three different types of norms were examined---the $\max$ norm, the KS norm, and the $1_{p}$ norm. The max norm was recommended; however, it was shown to produce system-level discrepancy functions which are nonsmooth. A cutting-plane algorithm and a move-limit strategy were developed and presented which adequately deal with the non-smoothness of the discrepancy functions.

The cutting-plane algorithm was tested on several hub frame examples. The algorithm tends to move deep into the infeasible region and work its way back to a feasible design. This process required about ten iterations regardless of the number of members (disciplines), where an iteration consists of a single call to each discipline for analysis and optimization. Additional cycles of the cutting plane algorithm may be executed with an accompanying reduction in move limit ranges. This results in a sequence of feasible designs at the end of each cycle which generally, though not strictly, converge toward optimality. Finally, it was observed that for optimization-intensive problems such as hub frame problems, the multilevel approach can reduce computation time over the single-level approach as the problem size increases even without exploiting the concurrent execution opportunity.

Future research may be directed towards improving the robustness of the cutting plane algorithm and move limit strategy. Another research need is to test the cutting plane algorithm on truly multidisciplinary problems.

\section{References}

Balling, R. J.; Sobieszczanski-Sobieski, J. 1994: Optimization of Coupled Systems: A Critical Overview of Approaches. AIAA Paper No. 94-4330, 5th ALAA/USAF/NASA/ISSMO Symposium on Multidisciplinary Analysis and Optimization, Panama City, FL, September 7-9, 1994.

Barthelemy, J-F. M.; Riley, M. F. 1988: Improved Multilevel Optimization Approach for Design of Complex Engineering Systems. AIAA Journal 26(3), 353-360.

Barthelemy, J-F. M.; Sobieszczanski-Sobieski, J. 1983: Optimum Sensitivity Derivatives of Objective Functions in Nonlinear Programming. ALAA Journal 21(6), 913-915. 
Grossman, B; Haftka, R. T.; Kao, P.-J.; Polen, D. M.; Rais-Rohani, M; Sobieszczanski-Sobieski, J. 1989: Integrated Aerodynamic Structural Design of a Transport Wing. ALAA Paper 89-2129, ALAA/AHS/ASEE Aircraft Design, Systems and Operations Conference, Seattle, WA, August 31-September 2, 1989.

Haftka, R. T.; Sobieszczanski-Sobieski, J.; Padula, S. L. 1992: On Options for Interdisciplinary Analysis and Design Optimization. Structural Optimization 4(2), 65-74.

Hajela, P.; Bloebaum, C. L.; Sobieszczanski-Sobieski, J. 1990: Application of Global Sensitivity Equations in Multidisciplinary Aircraft Synthesis. ALAA J. of Aircraft 27(12), 1002-1010.

Kelley, J. E. 1960: The Cutting-Plane Method for Solving Convex Programs. J. of the SIAM 8(4), 703-712.

Kreisselmeier, G.; Steinhauser, R. 1983: Application of Vector Performance Optimization to a Robust Control Loop Design for a Fighter Aircraft. Int'l J. of Control 37, 251-284.

Schmit, L. A.; Ramanathan, R. K. 1973: Multilevel Approach to Minimum Weight Design Including Buckling Constraints. AlAA Journal 16, 97-104.

Sobieszczanski-Sobieski, J. 1982: A Linear Decomposition Method for Large Optimization Problems--Blueprint for Development. NASA TM 83248.

Sobieszczanski-Sobieski, J. 1993: Two Alternative Ways for Solving the Coordination Problem in Multilevel Optimization. Structural Optimization 6, 205-215.

Sobieszczanski-Sobieski, J.; Bloebaum, C. L.; Hajela, P. 1991: Sensitivity of Control-Augmented Structure Obtained by a System Decomposition Method. AIAA Journal 29(2), 264-270.

Hareja, R; Haftka, R. T. 1986: Numerical Difficulties Associated with Using Equality Constraints to Achieve Multi-Level Decomposition in Structural Optimization. ALAA Paper 86-0854, ALAA/ASME/ASCE/AHS 27th Structures, Structural Dynamics, and Materials Conference, San Antonio, TX, April, 1986. 


\section{Appendix: Computational Details of Hub Frame Constraints}

\section{Displacement Constraints at Node P:}

$$
\begin{aligned}
& \delta / \delta_{\mathrm{a}}-1 \leq 0 \quad \theta / \theta_{\mathrm{a}}-1 \leq 0 \\
& \delta=\text { resultant translational displacement } \\
& \theta=\text { rotational displacement }
\end{aligned}
$$

$\delta_{\mathrm{a}} \quad \theta_{\mathrm{a}}$

$\begin{array}{lll}\text { two-member hub } & 0.249 \mathrm{~cm} & 0.0234 \mathrm{rad} \\ \text { eight-member hub } & 0.2 \mathrm{~cm} & 0.01 \mathrm{rad} \\ \text { twenty-member hub } & 0.2 \mathrm{~cm} & 0.01 \mathrm{rad}\end{array}$

\section{Stress Constraints:}

Normal and shear stresses ( $\sigma$ and $\tau$ ) were evaluated within the cross section at the top and bottom extreme fibers, at the centroid, and at the top and bottom of the web. This was done at both ends of the member except for the centroidal stresses which are constant along the length of the member. The following stress constraint was imposed at each location:

$\sigma_{e q} / \sigma_{a}-1 \leq 0$

$\sigma_{e q}=$ von Mises-Huber equivalent stress $=\left(\sigma^{2}+3 \tau^{2}\right)^{1 / 2}$

$\sigma_{\mathrm{a}}=$ allowable stress $=25 \mathrm{kN} / \mathrm{cm}^{2}$

\section{In-Plane Buckling Constraint:}

$$
\begin{aligned}
& \mathrm{N} / \mathrm{N}_{\mathrm{cr}}-1 \leq 0 \\
& \mathrm{~N}=\text { axial force (compression positive) } \\
& \mathrm{N}_{\mathrm{cr}}=2.05 \pi^{2} \mathrm{EI}_{\mathrm{xx}} / \mathrm{L}^{2} \\
& \mathrm{E}=\text { modulus of elasticity }=20,000 \mathrm{kN} / \mathrm{cm}^{2} \\
& I_{\mathrm{xx}}=\text { strong axis moment of inertia } \\
& \mathrm{L}=\text { member length }
\end{aligned}
$$


Out-of-Plane / Lateral-Torsional Buckling Constraint (at each end):

$$
\begin{aligned}
&\left(N / N_{c}\right)+\left(M / M_{c r}\right)^{1.75}-1 \leq 0 \\
& N=\text { axial force (compression positive) } \\
& M=\text { magnitude of bending moment } \\
& N_{c r}=2.05 \pi^{2} E_{y y} / L^{2} \\
& M_{c r}=\pi\left(E I_{y y} \mathrm{GI}_{z z}\right)^{1 / 2} / L \\
& E=\text { modulus of elasticity }=20,000 \mathrm{kN} / \mathrm{cm}^{2} \\
& G=\text { shear modulus of elasticity }=\mathrm{E} / 2(1+v) \\
& v=\text { Poisson's ratio }=0.3 \\
& I_{y y}=\text { weak axis moment of inertia } \\
& I_{z}=\text { torsional moment of inertia }=b_{1} t_{1}{ }^{3}+b_{2} t_{2}{ }^{3} \\
&+\left(h-t_{1}-t_{2}\right) b_{3}{ }^{3} \\
& L= \text { member length }
\end{aligned}
$$

Local Flange and Web Buckling Constraints (at each end):

$$
\begin{aligned}
& \sigma / \sigma_{\mathrm{cr}}+\left(\tau / \tau_{\mathrm{c}}\right)^{2}-1 \leq 0 \\
& \sigma=\text { normal stress (compression positive) } \\
& \tau=\text { shear stress }
\end{aligned}
$$

$\mathbf{\sigma}$

$\begin{array}{lll}\text { flanges } & \text { extreme fiber } & \text { mid-flange } \\ \text { web } & \text { mid-web } & \text { centroid }\end{array}$

$\sigma_{\mathrm{cr}}$

top flange

bottom flange

web

$$
\begin{array}{ll}
0.41 \mathrm{E}\left(2 \mathrm{t}_{1} / \mathrm{b}_{1}\right)^{2} & 0.55 \mathrm{E}\left(2 \mathrm{t}_{1} / \mathrm{b}_{1}\right)^{2} \\
0.41 \mathrm{E}\left(2 \mathrm{t}_{2} / \mathrm{b}_{2}\right)^{2} & 0.55 \mathrm{E}\left(2 \mathrm{t}_{2} / \mathrm{b}_{2}\right)^{2} \\
3.60 \mathrm{E}\left(\mathrm{b}_{3} /\left(\mathrm{h}-\mathrm{t}_{1}-\mathrm{t}_{2}\right)\right)^{2} & 4.80 \mathrm{E}\left(\mathrm{b}_{3} /\left(\mathrm{h}-\mathrm{t}_{1}-\mathrm{t}_{2}\right)\right)^{2}
\end{array}
$$

$\mathrm{E}=$ modulus of elasticity $=20,000 \mathrm{kN} / \mathrm{cm}^{2}$ 
Bounds on Section Variables:

$\begin{array}{llc} & \text { lower } & \text { upper } \\ \mathrm{b}_{1} & 2.0 \mathrm{~cm} & 6.0 \mathrm{~cm} \\ \mathrm{t}_{1} & 0.1 \mathrm{~cm} & 1.0 \mathrm{~cm} \\ \mathrm{~b}_{2} & 2.0 \mathrm{~cm} & 6.0 \mathrm{~cm} \\ \mathrm{t}_{2} & 0.1 \mathrm{~cm} & 1.0 \mathrm{~cm} \\ \mathrm{~b}_{3} & 0.1 \mathrm{~cm} & 1.0 \mathrm{~cm} \\ \mathrm{~h} & 3.0 \mathrm{~cm} & 8.0 \mathrm{~cm} \\ \mathrm{~A} & 0.68 \mathrm{~cm}^{2} & 10.00 \mathrm{~cm}^{2} \\ \mathrm{I} & 1.00 \mathrm{~cm}^{4} & 100.0 \mathrm{~cm}^{4}\end{array}$

Function Scaling Factors:

frame volume:

area coupling constraint:

moment of inertia coupling constraint:
$1000 \mathrm{~cm}^{3}$

$9.0 \mathrm{~cm}^{2}$

$99.0 \mathrm{~cm}^{4}$ 
Table 1: Variables and Functions for Two-Member Hub

\begin{tabular}{|c|l|}
\hline $\begin{array}{c}\text { Variable or } \\
\text { Function }\end{array}$ & \multicolumn{1}{|c|}{ Content } \\
\hline \hline $\mathrm{x}:$ & empty set \\
\hline $\mathrm{x}_{1}:$ & empty set \\
\hline $\mathrm{x}_{2}:$ & $\left\{\mathrm{b}_{1}, \mathrm{~b}_{2}, \mathrm{~b}_{3}, \mathrm{t}_{1}, \mathrm{t}_{2}, \mathrm{~h}\right.$ for Member PQ $\}$ \\
\hline $\mathrm{x}_{3}:$ & $\left\{\mathrm{b}_{1}, \mathrm{~b}_{2}, \mathrm{~b}_{3}, \mathrm{t}_{1}, \mathrm{t}_{2}, \mathrm{~h}\right.$ for Member $\left.\mathrm{PR}\right\}$ \\
\hline $\mathrm{g}_{1}:$ & $\{$ displacement constraints at Node $\mathrm{P}\}$ \\
\hline $\mathrm{g}_{2}:$ & $\begin{array}{l}\{\text { stress and buckling constraints for Member } \\
\text { PQ }\}\end{array}$ \\
\hline $\mathrm{g}_{3}:$ & $\{$ stress and buckling constraints for Member \\
\hline $\mathrm{f}_{1}:$ & PR $\}$ \\
\hline $\mathrm{f}_{2}:$ & $\{$ volume of the entire hub $\}$ \\
\hline $\mathrm{f}_{3}:$ & empty set \\
\hline $\mathrm{y}_{12}:$ & empty set \\
\hline $\mathrm{y}_{13}:$ & $\{\mathrm{N}, \mathrm{M}, \mathrm{V}$ for Member PQ for each loading case $\}$ \\
\hline $\mathrm{y}_{21}:$ & $\{$ A, I for Member PQ $\}$ \\
\hline $\mathrm{y}_{23}:$ & empty set \\
\hline $\mathrm{y}_{31}:$ & $\{$ A, I for Member PR $\}$ \\
\hline $\mathrm{y}_{32}:$ & empty set \\
\hline
\end{tabular}




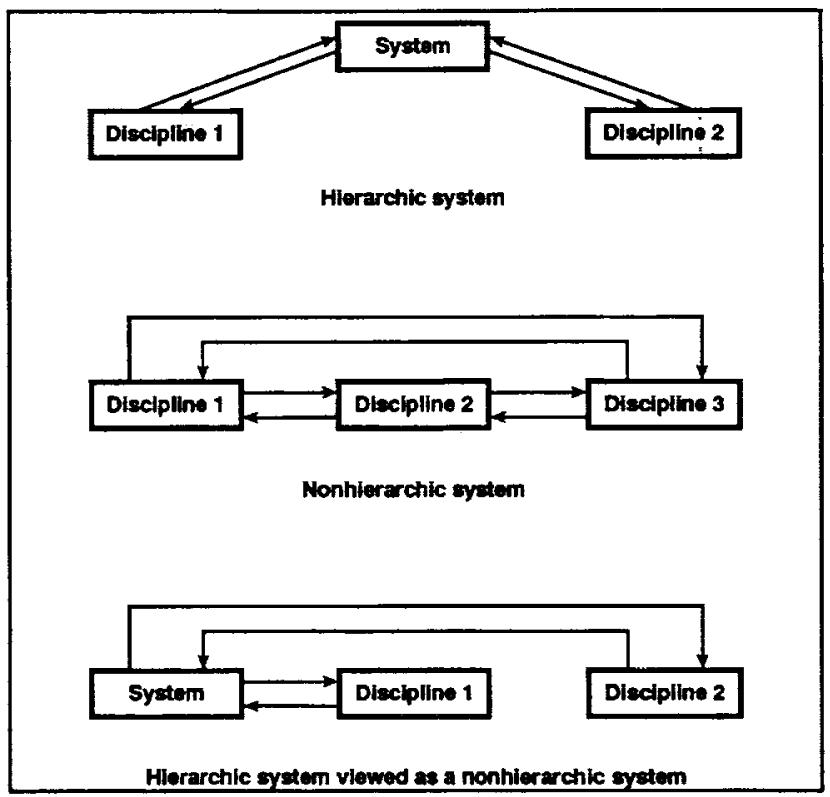

Fig. 1. Hierarchic and Nonhierarchic Systems.

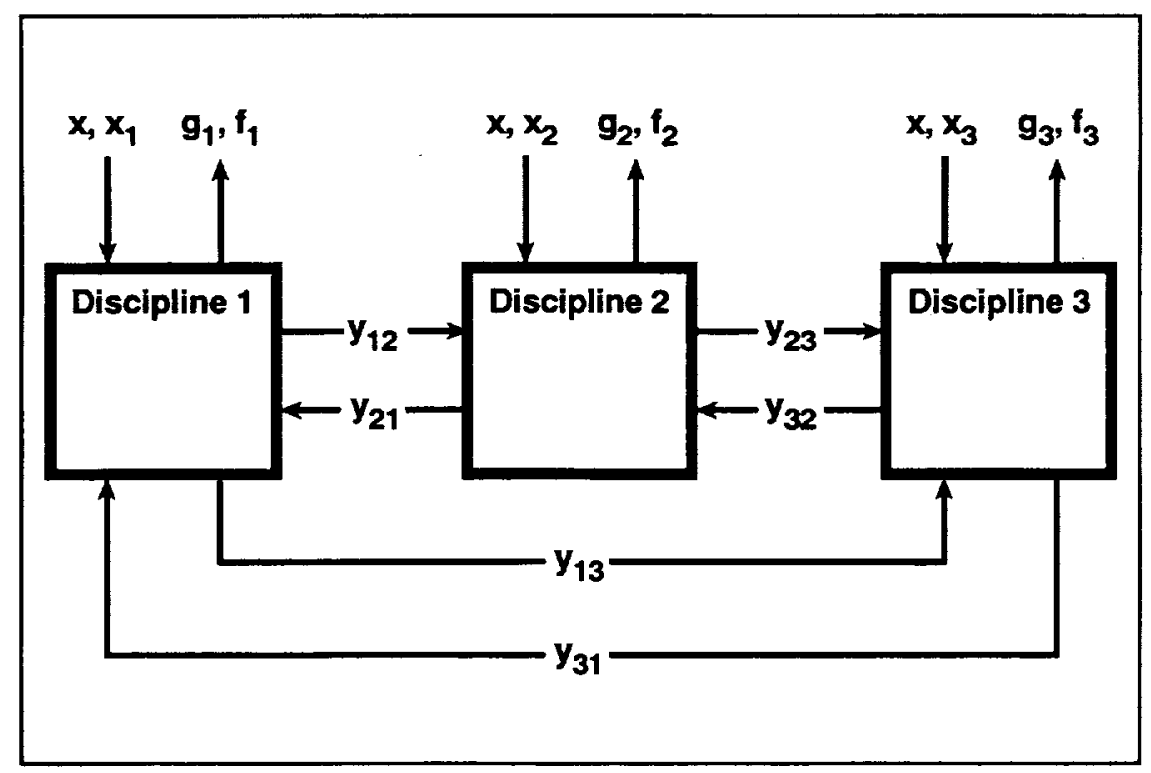

Fig. 2. Three-Discipline Coupled System. 


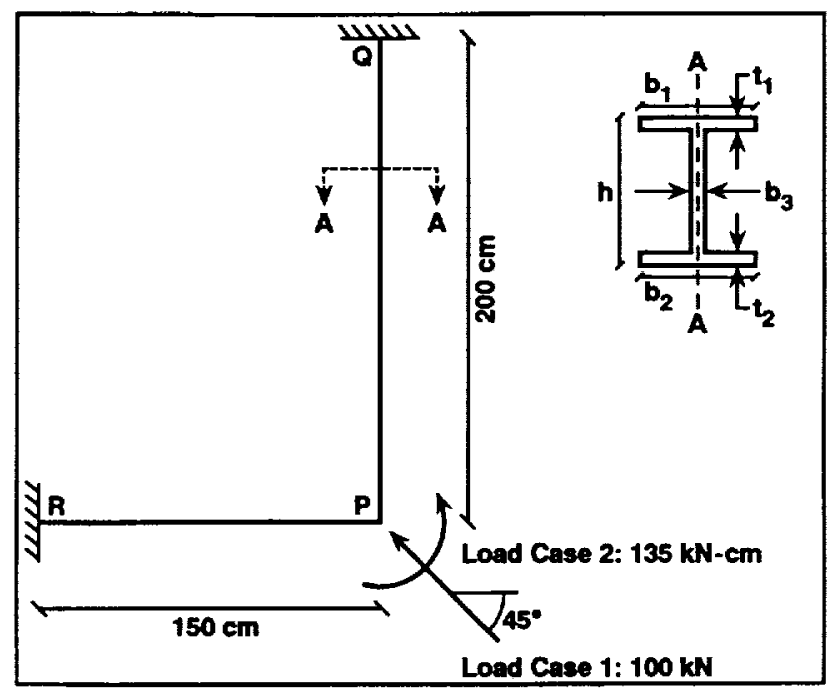

Fig. 3. Two-Member Hub Frame.

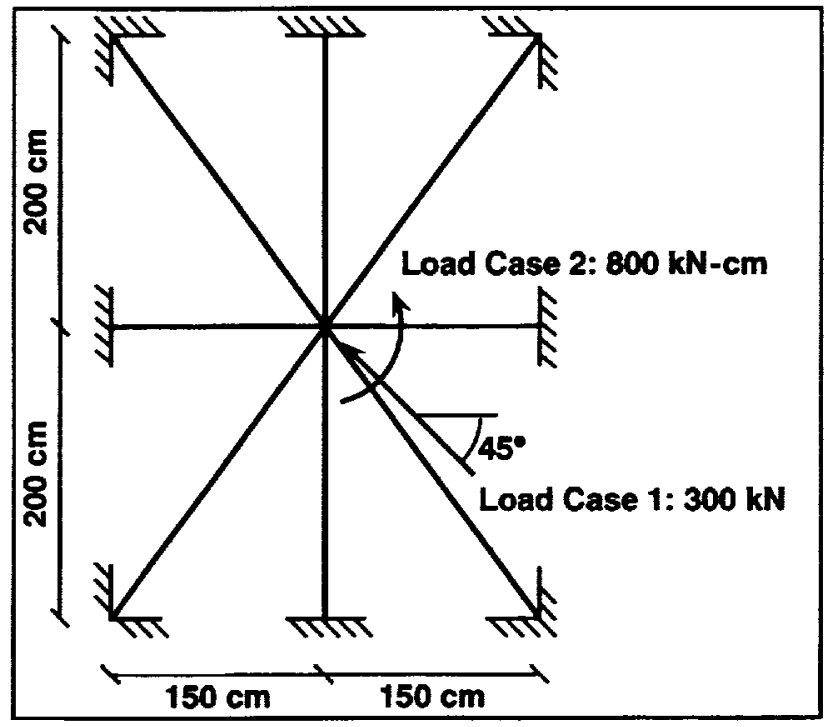

Fig. 4. Eight-Member Hub Frame. 


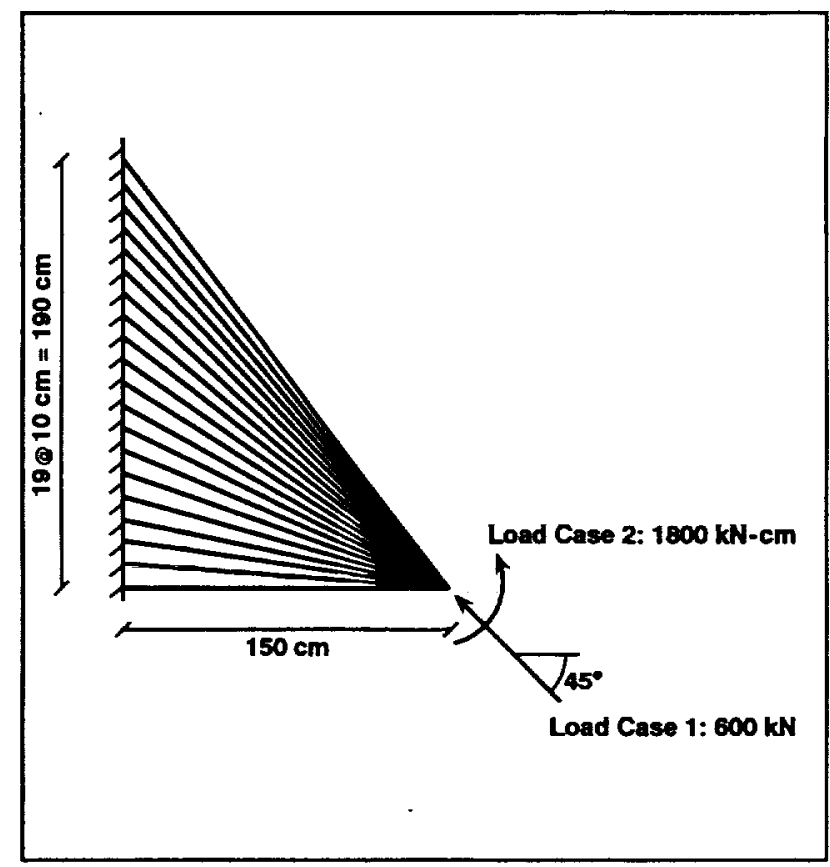

Fig. 5. Twenty-Member Hub Frame.

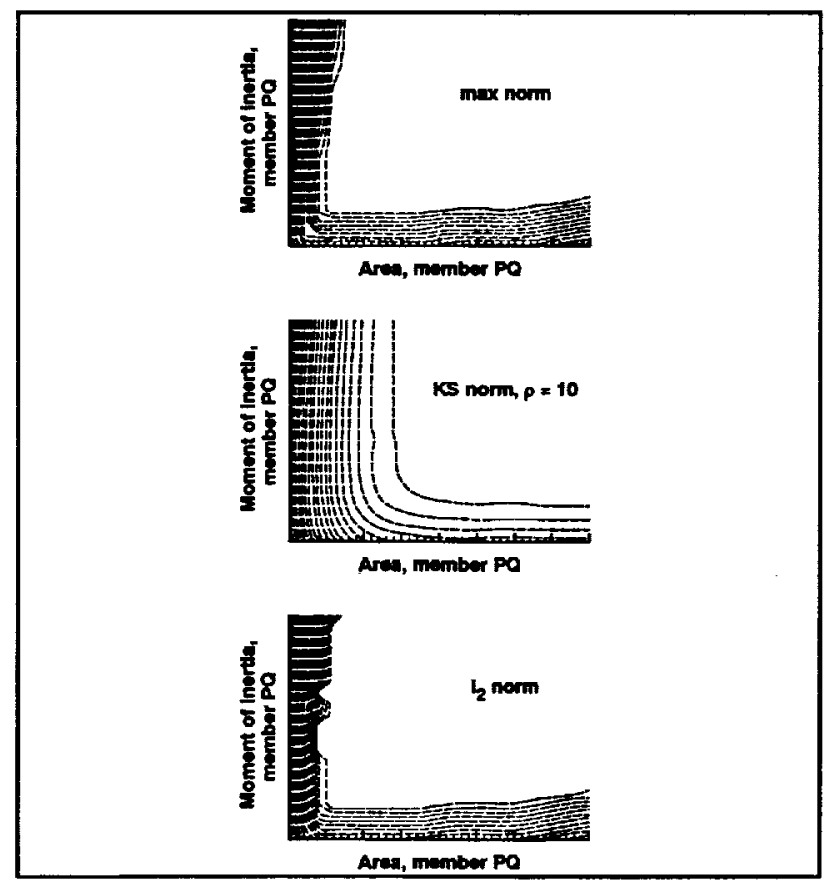

Fig. 6. Contour Plots of Discrepancy Function. 


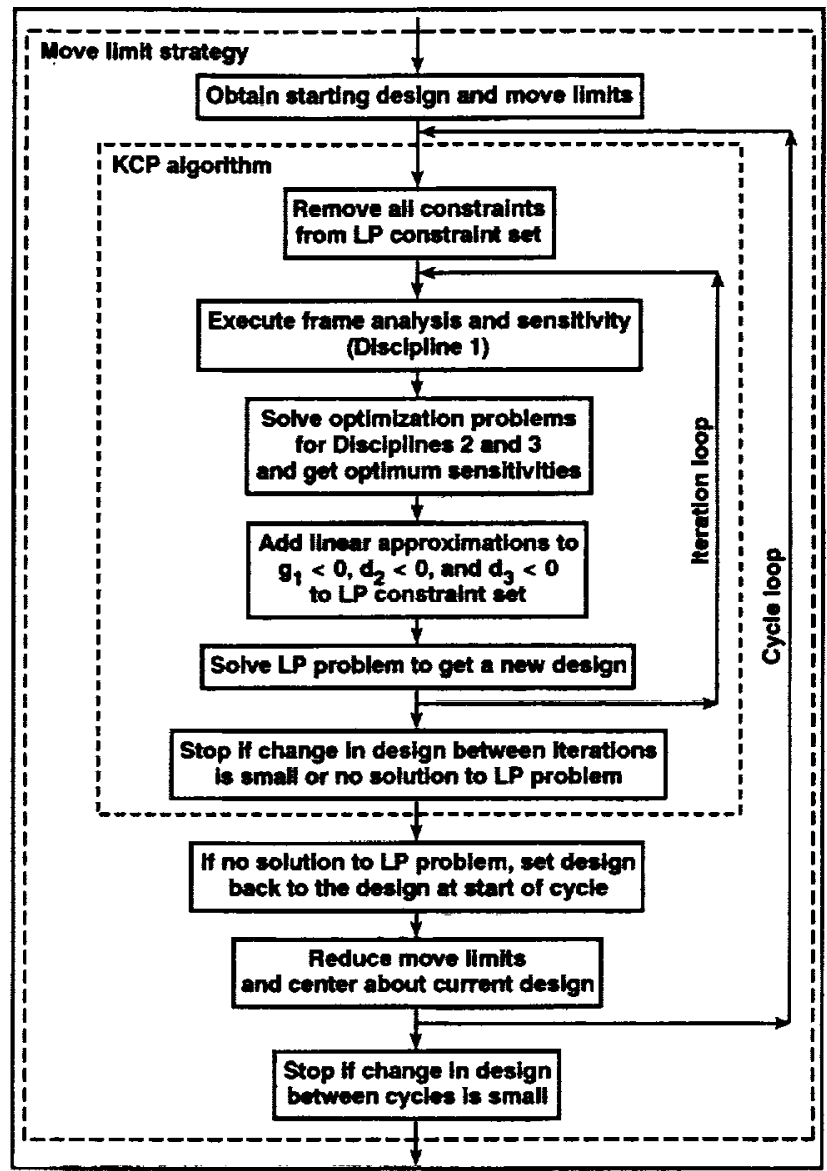

Fig. 7. KCP Algorithm and Move Limit Strategy.

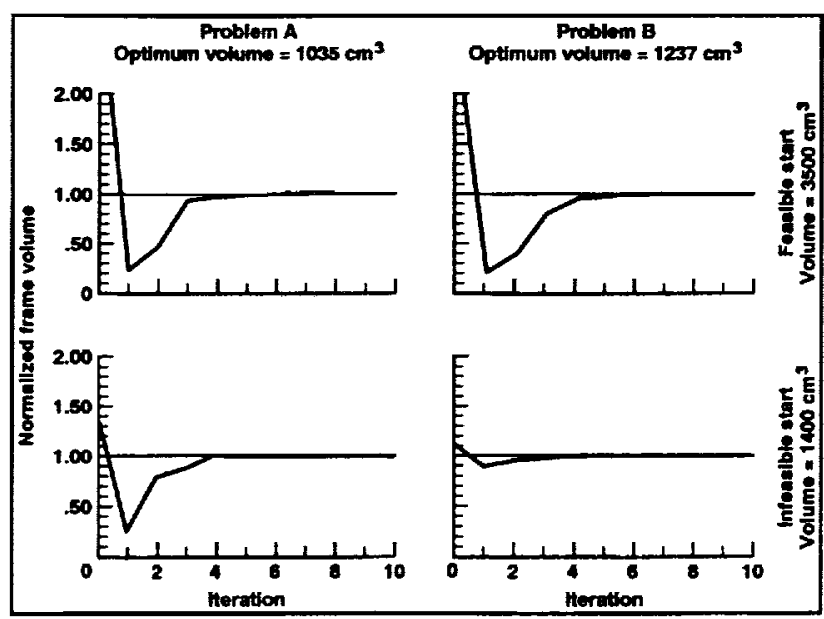

Fig. 8. Results for Two-Member Hub. 


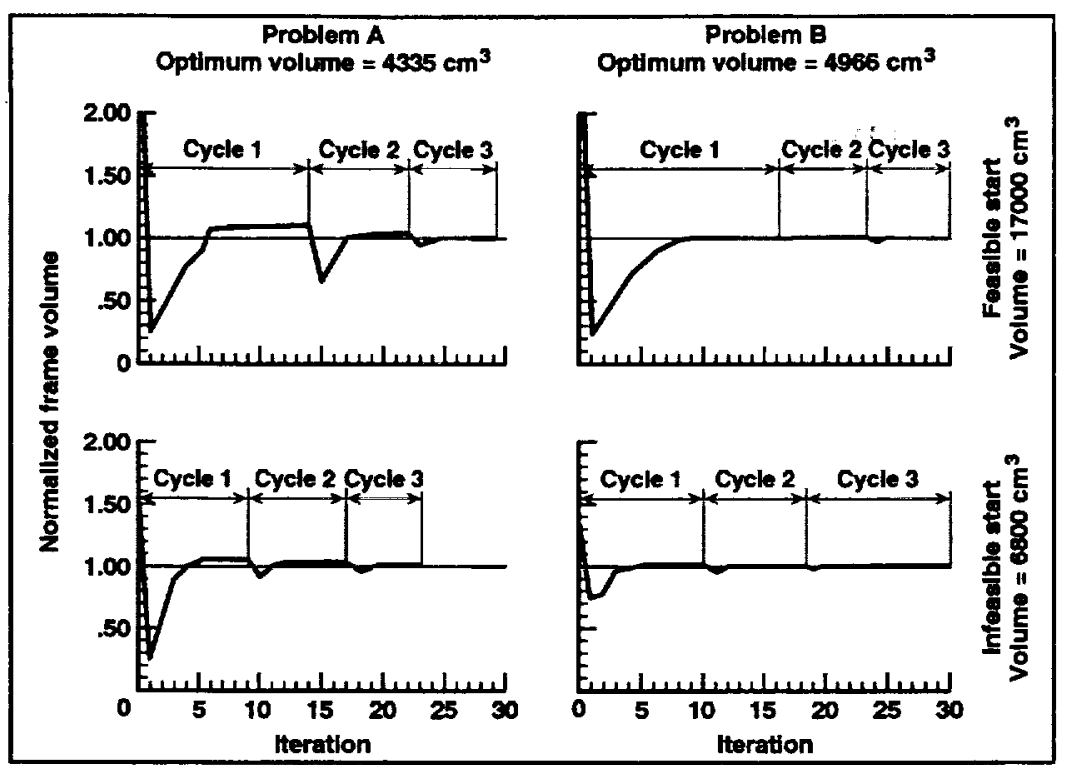

Fig. 9. Results for Eight-Member Hub.

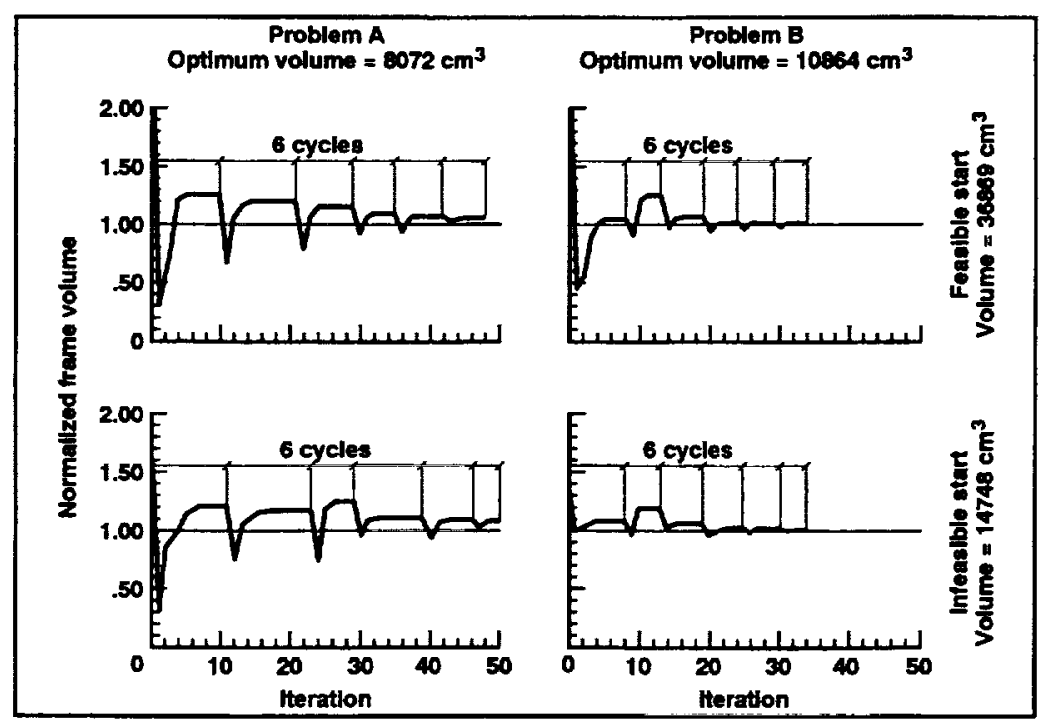

Fig. 10. Results for Twenty-Member Hub. 

Public reporting burden for this collection of information is estimated to average 1 hour per response. including the time for reviewing instructions, searching existing data sources, gathering and maintaining the data needed, and completing and reviewing the collection of information. Send comments regarding this burden estimate or any other aspect of this Davis Highway, Suite 1204, Arlington, VA 22202-4302, and to the Office of Management and Budget, Paperwork Reduction Project (0704-0188), Washington, DC 20503.

\begin{tabular}{|l|l|l|}
\hline 1. AGENCY USE ONLY(Leave blank) & $\begin{array}{l}\text { 2. REPORT DATE } \\
\text { December } 1994\end{array}$ & $\begin{array}{l}\text { 3. REPORT TYPE AND DATES COVERED } \\
\text { Contractor Report. }\end{array}$ \\
\hline
\end{tabular}

4. TITLE AND SUBTITLE

AN ALGORITHM FOR SOLVING THE SYSTEM-LEVEL PROB-

LEM IN MULTILEVEL OPTIMIZATION

C NAS1-19480

WU 505-90-52-01

6. AUTHOR(S)

R. J. Balling

J. Sobieszczanski-Sobieski

7. PERFORMING ORgANIZATION NAME(S) AND ADDRESS(ES)

Institute for Computer Applications in Science

and Engineering

Mail Stop 132C, NASA Langley Research Center

5. FUNDING NUMBERS

Hampton, VA 23681-0001

9. SPONSORING/MONITORING AGENCY NAME(S) AND ADDRESS(ES)

National Aeronautics and Space Administration

Langley Research Center

Hampton, VA 23681-0001

8. PERforming organization REPORT NUMBER

ICASE Report No. 94-96

10. SPONSORING/MONITORING AGENCY REPORT NUMBER

NASA CR-195015

ICASE Report No. 94-96

11. SUPPLEMENTARY NOTES

Langley Technical Monitor: Michael F. Card

Final Report

Proceedings of AIAA/USAF/NASA/ISSMO Symposium on Multidisciplinary Analysis and Optimization

12a. DISTRIBUTION/AVAILABILITY STATEMENT 12b. DISTRIBUTION CODE

Unclassified-Unlimited

Subject Category 64

13. ABSTRACT (Maximum 200 words)

A multilevel optimization approach which is applicable to nonhierarchic coupled systems is presented. The approach includes a general treatment of design (or behavior) constraints and coupling constraints at the discipline level through the use of norms. Three different types of norms are examined-the max norm, the Kreisselmeier-Steinhauser (IS) norm, and the $1_{p}$ norm. The max norm is recommended. The approach is demonstrated on a class of hub frame structures which simulate multidisciplinary systems. The max norm is shown to produce system-level constraint functions which are non-smooth. A cutting-plane algorithm is presented which adequately deals with the resulting corners in the constraint functions. The algorithm is tested on hub frames with increasing number of members (which simulate disciplines), and the results are summarized.

14. SUBJECT TERMS

Multidisciplinary; Optimization; multilevel

15. NUMBER OF PAGES

16. PRICE CODE

25

16. PRICE CODE

17. SECURITY CLASSIFICATION OF REPORT

Unclassified

SN 7540-01-280-5500
18. SECURITY CLASSIFICATION 19. SECURITY CLASSIFICATION OF THIS PAGE Unclassified OF ABSTRACT
20. LIMITATION OF ABSTRACT 
